\title{
Langue villeuse
}

\author{
Eric Burge BMus, Siddharth Kogilwaimath MD
}

- Citation : CMAJ 2021 April 19;193:E561. doi : 10.1503/cmaj.201559-f

Voir la version anglaise de l'article ici : www.cmaj.ca/lookup/doi/10.1503/cmaj.201559

$\mathbf{U}$

ne couche d'aspect villeux est apparue sur la langue d'un homme de 55 ans, après qu'il a passé 1 mois aux soins intensifs pour le syndrome de GuillainBarré. Durant son séjour à l'hôpital, il avait été intubé pendant 11 jours et avait subi une trachéotomie. Outre la détresse causée par l'apparence de sa langue, il était préoccupé par la diminution de son sens du goût. Le patient ne s'est pas plaint d'autres maux. Il avait des antécédents de tabagisme de 30 paquets-année. Aux soins intensifs, il avait reçu la combinaison pipéracilline-tazobactam, la combinaison triméthoprime-sulfaméthoxazole, de la ciprofloxacine et de la quétiapine. L'équipe soignante a d'abord diagnostiqué une candidose buccale, pour laquelle elle a prescrit plusieurs traitements de nystatine orale et de fluconazole systémique. L'absence de réponse aux antifongiques ainsi que l'aspect clinique typique de la langue et les antécédents du patient nous ont amenés à diagnostiquer une langue villeuse (figure 1).

La langue villeuse est une affection bénigne résultant de l'allongement des papilles filiformes en raison d'une accumulation de kératine. Elle peut être due à une exfoliation inadéquate (notamment attribuable à une diminution de l'alimentation orale, à une mauvaise hygiène buccale ou à une sécheresse buccale liée à la prise de médicaments anticholinergiques) et à une accélération de la kératinisation (comme c'est parfois le cas en présence d'une irritation liée au tabagisme) $)^{1}$. La langue villeuse est caractérisée par la présence d'une couche d'aspect chevelu sur le dos de la langue, les côtés et l'extrémité n'étant pas touchés ${ }^{2}$. Cette couche peut être de couleur crème, brune ou noire, dépendamment des facteurs extrinsèques, comme le régime alimentaire et le tabagisme, et des facteurs intrinsèques, comme les bactéries chromogènes et les champignons $s^{1,3}$.

La langue villeuse est généralement asymptomatique. Bien que le diagnostic soit clinique, une biopsie de la langue est parfois nécessaire pour clarifier le diagnostic si la lésion ne répond pas à un traitement conservateur ${ }^{1}$. Comme ce fut le cas chez notre patient, la langue villeuse est souvent confondue avec une candidose pseudomembraneuse et traitée de manière inefficace avec un médicament antifongique ${ }^{3}$. Le traitement consiste principalement en un débridement avec une brosse à poils doux; le patient doit également être rassuré ${ }^{1}$. Les agents aggravants doivent être éliminés et les facteurs de risque, modifiés, dans la mesure du possible. Le plus souvent, la langue villeuse se résorbe spontanément.

Nous pensons que la langue villeuse de notre patient était due à une période prolongée d'alimentation orale
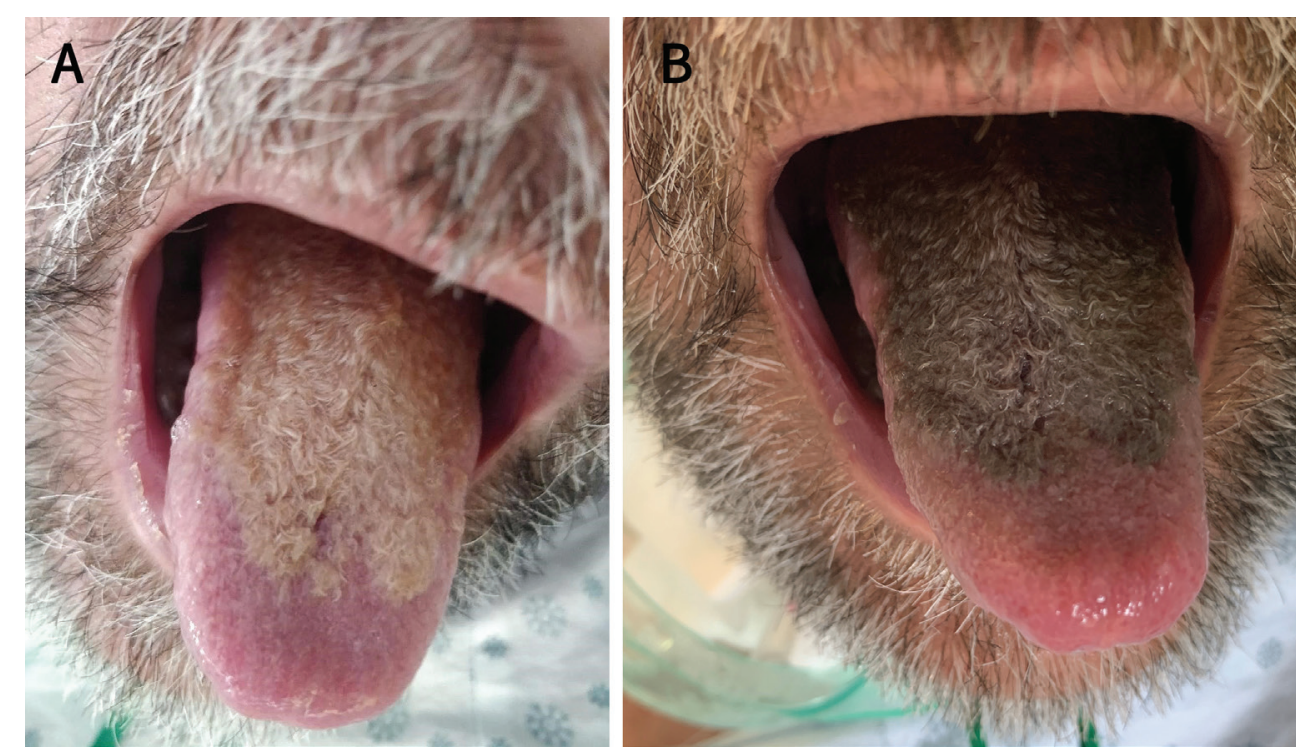

Figure 1 : Photographies d'un homme de 55 ans présentant une langue villeuse. A) Couche d'aspect villeux sur le dos de la langue après 1 mois aux soins intensifs. B) Quatre jours plus tard, la couleur de la couche a changé de façon marquée, après que le patient soit passé de l'alimentation par sonde à la prise orale, et du fluconazole à la ciprofloxacine. 
limitée, à une xérostomie liée aux effets anticholinergiques de la quétiapine et à une modification de la flore buccale due à divers traitements antimicrobiens. La langue du patient s'est considérablement améliorée après 2 mois.

\section{Références}

1. Gurvits GE, $\tan$ A. Black hairy tongue syndrome. World J Gastroenterol 2014;20:10845-50.

2. Mainville GN. Non-HPV papillary lesions of the oral mucosa: clinical and histopathologic features of reactive and neoplastic conditions. Head Neck Pathol 2019;13:71-9.

3. Schlager E, St. Claire C, Kurt C, et al. Black hairy tongue: predisposing factors, diagnosis, and treatment. Am J Clin Dermatol 2017;18:563-9.

Intérêts concurrents : Aucun déclaré.

Cet article a été révisé par des pairs.

Les auteurs ont obtenu le consentement du patient.

Affiliations : Collège de médecine (Burge); Division des maladies infectieuses, Faculté de médecine (Kogilwaimath), Université de la Saskatchewan, Saskatoon, Sask.

Propriété intellectuelle du contenu : Il s'agit d'un article en libre accès distribué conformément aux modalités de la licence Creative Commons Attribution (CC BY-NC-ND 4.0), qui permet l'utilisation, la diffusion et la reproduction dans tout médium à la condition que la publication originale soit adéquatement citée, que l'utilisation se fasse à des fins non commerciales (c.-à-d., recherche ou éducation) et qu'aucune modification ni adaptation n'y soit apportée. Voir : https://creativecommons.org/licenses/ by-nc-nd/4.0/deed.fr.

Correspondance : Siddharth Kogilwaimath, sik738@usask.ca

Les images cliniques sont choisies pour leur caractère particulièrement intéressant, classique ou impressionnant. Toute soumission d'image de haute résolution claire et bien identifiée doit être accompagnée d'une légende aux fins de publication. On demande aussi une brève explication (300 mots maximum) de la portée éducative des images, et des références minimales. Le consentement écrit du patient au regard de la publication doit être obtenu avant la soumission. 